\title{
Trayectorias escolares y estrategias de permanencia entre jóvenes indígenas universitarios (Salta, Argentina)
}

\section{Nuria Macarena Rodríguez y y María Macarena Ossola²}

1 Instituto de Investigaciones en Ciencias Sociales y Humanidades (ICSOH-UNSa-CONICET), Salta, Argentina.
(iD) https://orcid.org/oooo-0003-3692-294X Correo electrónico: nuria.macarena.rodriguez@gmail.com

2 Instituto de Investigaciones en Ciencias Sociales y Humanidades (ICSOH-UNSa-CONICET), Salta, Argentina. (iD) https://orcid.org/oooo-0oo1-7222-7464

Correo electrónico: macossola@gmail.com
Recibido:

18 de marzo de 2021

Aceptado:

28 de septiembre de 2021

doi: 10.34096/runa.v43i1.9965

Palabras clave

Jóvenes indígenas; Trayectorias escolares; Universidad;

Permanencia; Inclusión

\section{School trajectories and permanence strategies among young indigenous university students (Salta, Argentina)}

\begin{abstract}
This article presents an analysis of the permanence strategies deployed by indigenous youth during the development of their school careers from primary level to higher university level. The interest is placed in understanding how previous experiences, in formal education, permeate and condition their ways of staying at the University. In the development of work, the experiences and traces that indigenous university students bring with them from their years of schooling are recognized, not only with regard to school content, but also with regard to the construction of identifications and expectations.
\end{abstract}

Key wards

Indigenous youth; School trajetories; University; Permanence; Inclusion 


\section{Trajetórias escolares e estratégias de permanência de jovens universitários indígenas (Salta, Argentina)}

\section{Resumo}

Palavras-chave

Jovens indígenas; Trajetórias educacionais; Universidades; Permanência; Inclusão
Este artigo apresenta uma análise das estratégias de permanência implantadas por jovens indígenas durante o desenvolvimento de suas carreiras escolares do ensino fundamental ao ensino superior. O interesse está colocado em compreender como as experiências anteriores, na educação formal, permeiam e condicionam as suas formas de permanência na Universidade. No desenvolvimento do trabalho, reconhecem-se as experiências e os traços que os universitários indígenas trazem dos anos de escolaridade, não só no que diz respeito aos conteúdos escolares, mas também no que diz respeito à construção de identificações e expectativas.

\section{Introducción}

El presente artículo presenta el análisis de las estrategias de permanencia desplegadas por jóvenes indígenas durante el desarrollo de sus trayectorias escolares desde el nivel primario y hasta el nivel superior universitario; con el foco puesto en este último. El interés está puesto en comprender de qué forma las experiencias previas, en la educación formal, permean y condicionan sus formas de permanecer en la Universidad Nacional de Salta (UNSa), teniendo en cuenta, además, la participación en el Proyecto de Tutorías con Estudiantes de Pueblos Originarios (ProTconPO), implementado por la casa de altos estudios desde 2010.

Según datos oficiales, para ese año existían en Argentina 955.032 personas que se reconocían pertenecientes o descendientes en primera generación de un pueblo indígena (INDEC, 2010), lo que equivalía al 2,3\% de la población nacional. No obstante esta cifra, en múltiples ámbitos sociales a nivel nacional (las instituciones de educación formal incluidas) aún tiene notable vigencia el imaginario social a través del cual se identifica a los argentinos con los descendientes de los inmigrantes europeos, con el cual se invisibiliza la diversidad cultural y lingüística existente.

De esta manera, los pueblos indígenas afrontan cotidianamente situaciones de discriminación y racismo, principalmente en el acceso a los sistemas de salud y educación (Anaya, 2012). Este último, si bien ha adecuado parcialmente los contenidos y las modalidades hacia un eje intercultural, aún cuenta con grandes dificultades para garantizar a los pueblos indígenas el ingreso, la permanencia y la finalización a la totalidad de los niveles escolares existentes: inicial, primaria, secundaria y superior (universitario y terciario).

Como hemos destacado anteriormente (Ossola, 2020), el acceso a mayores niveles de escolaridad representa, para estos pueblos, la adquisición de un derecho-clave o derecho-llave, que luego les permitirá acceder a otros derechos. Desde esta perspectiva, avanzar en la educación formal se vislumbra como una doble posibilidad. En primer lugar, supone una oportunidad personal para 
incrementar los conocimientos adquiridos, afianzar el uso de la lengua española (entre quienes tienen una lengua indígena como primera lengua, como es el caso de los wichí que se describe más adelante) y ampliar los vínculos con la sociedad mayoritaria. En segundo lugar, representa una modalidad de colaboración con las luchas y las demandas del movimiento indígena. Debido a ello, la escolaridad formal es percibida como una herramienta necesaria en un contexto de falta de oportunidades -especialmente para las y los jóvenes-y de opresión cultural, territorial y lingüística.

Es importante destacar que definimos a las trayectorias escolares como los recorridos de las personas en el sistema escolar, comparados con la expectativa que supone el diseño de tal sistema (Terigi, 2008). Para el caso en estudio, es importante señalar que entre los pueblos indígenas se evidencia un alejamiento pronunciado respecto de las trayectorias ajustadas a la normativa conforme los sujetos avanzan en edad, y más aún cuando son correlacionados la edad y el año cursado o, específicamente, la edad y el año aprobado. De este modo, la repitencia, la sobreedad y la deserción caracterizan las trayectorias escolares de los estudiantes indígenas en Argentina (UNICEF, 2009).

Con el estudio de las trayectorias buscamos poner en cuestión ciertas ideas sedimentadas sobre el fracaso escolar de los niños, adolescentes y jóvenes indígenas. En este sentido, otras investigaciones han mostrado que en la escuela se realizan relecturas globales de las experiencias individuales y colectivas previas (Aikman, 2003), a la vez que se organizan y proyectan alternativas de futuro (Czarny, 2008). La experiencia universitaria, en particular, reviste un espacio que permite reconstruir las huellas que la escolaridad ha dejado en estos pueblos (Luján, Soto y Rosso, 2018).

Nos referimos a las estrategias de permanencia desplegadas por las y los jóvenes indígenas universitarios como aquellas prácticas y actitudes que son asumidas durante el desarrollo de sus carreras. Estas les permiten transitar su formación superior en contextos que mayoritariamente se presentan como adversos (debido a las necesidades económicas y la distancia que supone ir a la ciudad y apartarse de las comunidades) y, como señalaremos más adelante, se vinculan tanto a la reelaboración de experiencias previas como así también a nuevos aprendizajes realizados en la ciudad y la universidad. Cabe destacar que las tácticas de permanencia se diferencian de aquellas políticas elaboradas institucionalmente para intentar favorecer una continuidad de los estudiantes en la universidad, las cuales representan "estrategias de retención" (Rodríguez, 2020).

En nuestro caso de estudio, abordamos las estrategias de permanencia que asumen aspectos tanto individuales como colectivos, ya que se producen en el marco de la Comunidad de Estudiantes Universitarios de Pueblos Originarios (CEUPO).

El CEUPO es un espacio en el que se han organizado estudiantes indígenas de la UNSa, y se define como:

Somos un grupo de estudiantes universitarios que lucha por el reconocimiento y el respeto a la diversidad cultural en nuestra Universidad. En este grupo participamos en forma voluntaria estudiantes de las diferentes facultades, compartiendo saberes, conocimientos y experiencias de nuestras culturas y la vida en la Universidad. Somos una comunidad que tiene voz, somos eco de nuestras culturas ancestrales, con identidad, con historia; somos una comunidad que se abre caminos en esta 
UNIVERSIDAD. (Presentación utilizada por CEUPO en varias actividades, tomada de su página de Facebook: https://www.facebook.com/CEUPO-Comunidad-deEstudiantes-Universitarios-de-Pueblos-Originarios-463746263836116/)

Este grupo se conforma por estudiantes que pertenecen a diversos pueblos indígenas. En las interacciones que hemos mapeado, encontramos que la convivencia entre ellos genera nuevas estrategias de permanencia en la institución, asentadas en diversas prácticas culturales, artísticas y políticas, entre otras.

\section{Metodología}

Este trabajo se inscribe en una lógica cualitativa de investigación. Buscamos comprender la realidad en estudio basándonos en las percepciones de las personas que la conforman, es decir, a partir de las voces de las y los estudiantes que participan de la investigación, y en interacción con nuestras propias significaciones. Principalmente utilizamos estrategias etnográficas, entre las cuales destacan las entrevistas semiestructuradas y en profundidad, que nos permiten reconstruir las perspectivas de los interlocutores en base a las connotaciones y significaciones que ellas mismas otorgan a su paso por diferentes instancias escolares.

Concebimos a la etnografía como una estrategia basada en la observación, la realización de entrevistas y la recolección de documentos que supone la estancia prolongada del investigador en el sitio donde se producen los procesos que intenta describir y analizar. De manera específica, "la etnografía tiene por objetivo dar cuenta del conocimiento local a través de la reconstrucción interpretativa de las relaciones sociales, los saberes y la cultura de un determinado grupo, institución o proceso social" (Levinson, Sandoval Flores y Bertely-Busquets, 2007, p. 825).

En cuanto a las entrevistas en profundidad, estas se orientan a reconstruir las trayectorias socioescolares a partir de narrativas biográficas. Para realizar esta reconstrucción se pone el foco en las intersecciones entre biografía y educación, que permiten reconocer la importancia que tienen las instituciones escolares en la (re)elaboración de los proyectos de vida de los sujetos. Esta decisión asume que -en educación- la investigación autobiográfica genera conocimientos sobre los sujetos, pero también sobre sus vínculos sociales y territoriales (DeloryMomberger, 2009).

El trabajo de campo se desarrolló en la Universidad Nacional de Salta entre 2015 y 2019, periodo en el cual pudimos acompañar diferentes actividades desarrolladas por el CEUPO, como así también por el Proyecto de Tutorías con Pueblos Originarios (ProTconPo). Este último se constituye como el espacio institucional creado para el acompañamiento pedagógico y afectivo de las y los estudiantes indígenas en la UNSa. Se basa principalmente en el apoyo a través de tutores-pares (estudiantes de grado) que cuentan con un cargo rentado para ejercer ese rol. En sus inicios, los puestos de tutores fueron mayoritariamente ocupados por no-indígenas, pero en los últimos años se observa cada vez a más estudiantes indígenas oficiando como tutores. Cabe aclarar que la participación, tanto en el CEUPO como en las tutorías, es opcional.

1. Los nombres de los y las estudiantes son ficticios, a fines de mantener su anonimato.
En este artículo recuperamos las voces de la docente que coordina el proyecto de tutorías; dos tutoras (Sara, ${ }^{1}$ perteneciente a una comunidad wichí y que estudia el Profesorado en Ciencias de la Educación, y Nilda, extutora 
no-indígena, que se graduó como Profesora en Ciencias de la Educación y aún colabora con las tutorías) y dos participantes de las tutorías: uno indígena y una no-indígena.

Cabe recordar que nuestro objetivo es comprender de qué forma las experiencias previas, en la educación formal, permean y condicionan sus formas de permanecer en la Universidad. Por ello, identificamos la manera en que algunos aspectos de esas dinámicas tienen continuidad en la universidad, otros se modifican y aparecen nuevos, configurando experiencias disímiles hacia el interior del CEUPO. Esto es relevante si se tiene en cuenta que las trayectorias educativas son productoras de subjetividades. En ese sentido, se reconocen las experiencias y huellas que los estudiantes universitarios traen consigo de sus años de escolarización, no solo en lo que refiere a contenidos escolares, sino también en lo atinente a la constitución de significaciones y expectativas.

\section{Experiencias en la educación primaria}

Desde la conformación del Estado Nacional, el nivel primario es el que ha recibido históricamente a los indígenas, pues el objetivo consistía en su integración a la identidad nacional, por lo que la escuela debía cumplir la función de castellanizarlos y transformar sus prácticas culturales (Hirsch y Serrudo, 2010). Las funciones atribuidas a esta institución han ido mutando, pero es importante resaltar -como aspecto inicial a tener en cuenta para analizar las experiencias en este nivel- el hecho de que es al que más -y desde hace más tiempo- acceden los estudiantes indígenas.

En nuestro país, como en América Latina en general, la tasa de escolaridad de los pueblos indígenas en el nivel primario ha alcanzado niveles similares a la tasa de escolaridad de la población total. Así pues, en Argentina, la absoluta mayoría de la población indígena de entre cinco y catorce años asiste a un establecimiento educativo $(94,6 \%)$, proporción similar a la total del país: 95,3\% (INDEC, 2004-2005).

Respecto de este nivel, es válido aclarar que varios de los miembros de pueblos indígenas realizan su educación primaria en escuelas rurales organizadas como plurigrado, ${ }^{2}$ que es recordada de manera positiva en los relatos de los y las estudiantes del CEUPO. Al respecto, Sara sostiene:

lo más lindo que me acuerdo de esa etapa de la primaria es que antes podía cursar o podía estar con todos los grados, no solamente niños, sino que se mezclaba con gente grande, con chicos que ya estaban en secundaria... y había ese cuidado de, de parte de todos ¿no? (Entrevista a extutora del ProTconPO, proveniente de una comunidad wichí)

Este pasaje permite observar que el valor otorgado al plurigrado no se sustenta en aspectos pedagógicos, sino afectivos. La modalidad del plurigrado suponía que los mayores "cuidaran" a los más pequeños. Además, algunos eran hermanos o primos, lo cual posibilitaba la extensión de los lazos familiares. Quizás allí los estudiantes encontraban una estrategia de permanencia, que tiene ciertas continuidades en el ProTconPo, ya que los estudiantes avanzados acompañan ("cuidan") a los ingresantes y a los de los primeros años. Esta modalidad también es mencionada por Marta en su narrativa biográfica: "tenía que nos juntaban, tercero y segundo estábamos juntos y nada... me acuerdo de la maestra y los compañeros de toda la gente, te conocés con todos en
2. En la mayoría de las escuelas rurales, la organización está dada por "plurigrados" en los que se “agrupa alumnos que están cursando distintos grados de su escolaridad primaria en forma simultánea con un mismo maestro" (Terigi, 2006, p. 4) 
la comunidad y tengo muy lindos recuerdos" (Entrevista a participante del ProTconPO, proveniente de una comunidad kolla).

Los recuerdos que destaca Marta incluyen al plurigrado como espacio de sociabilización común, de encuentro asociado a lo afectivo, pero no en relación con el "cuidado" como en el caso de Sara. El hecho de "conocerse todos" también es una estrategia de permanencia en el ProTconPo. La entrevistada comenta que los encuentros entre estudiantes de todas las facultades son favorecedores, en tanto compartir experiencias les permite "no sentirse solos".

Sin embargo, el plurigrado también puede tener valoraciones negativas. Sulca (2018), desde su propia experiencia, reconoce como una desventaja para la continuidad en el siguiente nivel del sistema educativo el inicio de este recorrido en una escuela rural, plurigrado, porque tiene como consecuencia el aprendizaje de contenidos mínimos, insuficientes. Resulta pertinente aclarar que no todos los niños indígenas asisten a escuelas plurigrado. Quienes provienen de pueblos más grandes no concurrieron a esta modalidad de escuelas rurales. De hecho, Marta, por ejemplo, migró desde su comunidad de origen (Colanzulí) al pueblo principal de la zona (Iruya) y allí cursó el último tramo de su primaria y toda la secundaria. Respecto de este cambio, expresa:

la primaria la terminé en Iruya, cierto, sí, también fue un cambio muy fuerte para mi familia y para mí. La escuela era distinta, éramos muchos más alumnos, tenía ya dos docentes, la escuela era mucho más grande y me acuerdo que sí me costó un poco adaptarme a la escuela. (Entrevista a Marta, participante del ProTconPO, proveniente de una comunidad kolla)

En este pasaje de la entrevista, la estudiante relata cómo el hecho de no conocer a las personas, a los compañeros y de ser más estudiantes tornó difícil su adaptación a la primaria. Además, en esta institución tenía compañeros "criollos", mientras que en su anterior escuela eran todos de la comunidad kolla. Esta es otra característica que difiere de una comunidad a otra, según la cercanía a pueblos o ciudades con poblaciones no-indígenas, y no es un dato menor, en tanto muchos de los estudiantes indígenas identifican sus primeras experiencias de discriminación por su identidad indígena con el ingreso a la educación formal. En relación con esto, Czarny (2008) explica cómo el acto de concurrir a la escuela ha tenido, para muchos indígenas, el costo de la negación de su identidad, como consecuencia de la discriminación allí vivida.

En diferentes conversaciones y encuentros con integrantes del CEUPO se ponen de manifiesto situaciones de discriminación que las y los jóvenes han vivido por ser "indios" o "coyas", y que acontecieron en contextos de educación formal. Sin embargo, estas mismas experiencias son resignificadas por quienes, lejos de ocultarse o negar su identidad indígena, la reivindican, constituyéndola como una de sus estrategias comunitarias de permanencia en la Universidad.

Es menester señalar que las escuelas en las que todos los estudiantes se autoidentifican indígenas no se encuentran ajenas a la discriminación. Al respecto, Sara nos cuenta:

Tal vez negativo, esto de no poder hablar el idioma en... eh... cuando ya éramos más grandes, la primaria, por ahí quinto grado o sexto, por temor a esto ¿no? De que siempre era hablar mal del profesor o del maestro. (Entrevista a Sara, extutora del ProTconPO, proveniente de una comunidad wichí) 
La lengua, como diacrítico de identidad, no escapa a las dinámicas de estigmatización y desconocimiento que se producen en los espacios educativos argentinos, aún en la actualidad (Ossola, 2015). Asimismo, las tensiones sobre las lenguas expresan problemáticas más amplias sobre los diferentes segmentos que componen la sociedad, y que se recrean y actualizan en ámbitos escolares.

Otro ejemplo de este tipo de dinámicas entre inclusión-exclusión en la educación primaria se encuentra en los manuales distribuidos por el Gobierno. En una de las actividades organizadas por el CEUPO, un estudiante indígena contaba que en el manual que le habían dado para estudiar se hablaba de los "aborígenes" en términos de pasado, como si ya no existieran. Es decir, les enseñaban a indígenas, que los indígenas ya no existían. Se refuerza la idea, muchas veces promovida por el Estado moderno, de habitantes preestatales, que representan el pasado y el atraso.

Aun habiendo sufrido la discriminación desde el nivel primario de educación, para quienes forman parte del ProTconPo la identidad indígena es motivo de orgullo y una estrategia de permanencia. Al respecto, Marta sostiene: "a pesar de que yo venía con una idea de que 'ésta es mi identidad y es quien soy', yo creo que el CEUPO me ayudó más a fortalecer todavía eso”. Así pues, lejos de tender a ocultar su identidad para pasar desapercibidos, se orientan a permanecer reivindicando esa identidad. Nos encontramos ante fenómenos en movimiento, en que las relaciones entre ocultamiento y reivindicación de la identidad indígena van mutando entre niveles escolares. Sulca (2018) elabora una valiosa explicación de este proceso, al describir en primera persona cómo se alejó de su identidad indígena durante la mayor parte de su trayectoria escolar para evitar la discriminación, pero el tránsito por la Universidad la cambió sustancialmente. De tal modo, reconoce que los procesos (de)formativos de la academia le permitieron alejarse del sufrimiento que relacionaba con su identidad indígena y, en ese sentido, reencontrarse de forma diferente con esta identidad que no había destruido, sino resguardado (Sulca, 2018).

\section{Experiencias en la educación secundaria}

Las trayectorias educativas se van consolidando en base a las experiencias en todos los niveles y, en caso de haber cortes que signifiquen cambios significativos, no siempre están dados por la división en niveles que se realiza desde lo normativo. Un ejemplo de ello es lo comentado en el apartado anterior respecto del cursado de la primaria de Marta; el quiebre, en ese caso, se produjo por una migración y no por un cambio de nivel educativo.

Los estudiantes del secundario, entonces, no son otros que los que vienen de haber vivido las experiencias de la primaria, de plurigrado, de discriminación, de estrategias afectivas, etc. Pero ingresan ahora a un nivel al que no muchos indígenas accedían previamente al año 2007 (con la puesta en vigencia de la Ley de Educación Nacional, que amplía la obligatoriedad al nivel secundario), lo que los convirtió en pioneros. Sumado a esto, la mayoría de ellos debe viajar para acceder a este nivel, pues existe menor cantidad de colegios secundarios que de escuelas primarias en áreas rurales, donde se ubican mayoritariamente las comunidades indígenas de Salta y Jujuy.

Sara nos cuenta que para cursar la secundaria debió mudarse a otra comunidad: 
después me fui a La Puntana, que queda a quince kilómetros de mi comunidad, porque en mi comunidad no había colegio, entonces hice toda mi secundaria en La Puntana. Bueno, ahí la diferencia es que en La Puntana hay como... la mayoría son wichí, entonces hablan perfecto el idioma. (Entrevista a Sara, extutora del ProTconPO, proveniente de una comunidad wichí).

Si bien debió trasladarse para poder continuar sus estudios, menciona cómo, a diferencia de su primaria, en la secundaria la lengua predominante era el wichí y eso le resultó favoreció su adaptación al nuevo entorno. En el relato de Sara se encuentra siempre presente la referencia al idioma con un valor positivo, lo cual coincide con lo señalado por Ossola y Hecht (2011) en cuanto a que entre los jóvenes wichí la lengua se liga a la afectividad y se constituye en la marca explícita de su pertenencia grupal.

En ese momento de la entrevista, la estudiante nos comenta que le sorprendió en la secundaria encontrarse con compañeros que no hablaban el español. De hecho, relata, uno de ellos ingresó a la UNSa hablando solo wichí: "uno de mis compañeros que él hizo toda la primaria en La Puntana, porque es de ahí, él decía que recién aprendió a hablar el español llegando acá a la Universidad" (Entrevista a Sara, extutora del ProTconPO, proveniente de una comunidad Wichí).

Aprender a hablar, a leer y a estudiar en español no es algo para lo que la Universidad esté pensada ni preparada, sin embargo, las experiencias nos muestran cómo los estudiantes indígenas despliegan estrategias en este sentido.

La cuestión de la lengua indígena también nos permite identificar estrategias de permanencia de los estudiantes universitarios del CEUPO, específicamente los wichí, cuya primera lengua es la homónima. Así, Sara nos comenta cómo procedían ante textos o palabras que no comprendían:

y tal vez la ventaja que... que tuve o tuvimos fue que vinimos en grupo y eso también nos ayudó un poco a poder hablarlo en el idioma, buscar ejemplos que puedan estar relacionados con nuestra cultura y... creo que eso también me ayudó, pero dificultades tuve, tuvimos bastantes. (Entrevista a Sara, extutora del ProTconPO, proveniente de una comunidad wichí)

Este fragmento y la forma en que Sara cuenta la experiencia que comparte con sus compañeros nos muestra que andar en grupo es una estrategia de permanencia muy importante para estos jóvenes. Al respecto, en trabajos anteriores (Ossola, 2015) reconocemos como una de las principales connotaciones de la lengua wichí la de constituirse como una herramienta para la unión grupal, que les permite a los estudiantes universitarios wichí resolver de forma colectiva el tránsito por un contexto nuevo y diferente a los espacios sociales conocidos.

En el caso de los wichí, entonces, andar en grupo está relacionado con el hecho de comunicarse en su lengua, como así también con la posibilidad de compartir las diferentes experiencias sociales por las que transitan. Esta última es una estrategia que se torna más general en todos los estudiantes del CEUPO e incluso se constituye en un aspecto importante para el trabajo de los tutores. De esta forma, reconocemos que andar en grupo constituye también una estrategia de retención, en tanto el ProTconPO como política institucional promueve las actividades grupales. 
Retomando la cuestión de las experiencias en los colegios secundarios, es interesante mencionar que notamos ciertas diferencias entre los docentes según su procedencia, es decir, en relación con su pertenencia o no a la comunidad. En términos generales, los relatos de los estudiantes indígenas demuestran mayor compromiso cuando los docentes son de la propia comunidad y menor cuando son "criollos" que van a enseñar a escuelas rurales, quienes suelen faltar, o concurrir y no cumplir con sus tareas.

En el caso de Sara, quien cursó en un secundario donde ningún docente era de la comunidad, su experiencia estuvo ligada a las ausencias: "No, no daban clase, porque bueno, también había docentes que se iban, tenían familiares entonces desaparecían una semana, volvían, entonces era como que no había tanto control" (Entrevista a Sara, extutora del ProTconPO, proveniente de una comunidad wichí).

En este pasaje refiere al ausentismo, que es un comentario recurrente en conversaciones informales: docentes que acuden a escuelas rurales para ingresar al sistema, se toman licencias médicas y luego piden traslados a las ciudades, o que, en sus últimos años de servicio concurren a trabajar a las comunidades para cobrar por zona desfavorable y así obtener una mejora en su haber jubilatorio, entre otras situaciones aludidas.

Pero las ausencias a las que referimos no están solo relacionadas con el ausentismo, sino que existen ausencias aún en la presencia física: "cómo los docentes [del secundario] trataban a los... a los padres o a los propios estudiantes, de que bueno, no hay que enseñarles tanto porque no van a seguir estudiando o no van a hacer una carrera universitaria ni terciario" (Entrevista a Sara, extutora del ProTconPO, proveniente de una comunidad Wichí).

Se encuentran, en la experiencia de Sara, cuestiones que dan cuenta de cómo los discursos y prácticas escolares están teñidas de representaciones negativas que se asientan en el origen social y cultural de los estudiantes, y se traducen en la ausencia de acompañamiento y formación, pues carecería -desde su punto de vista- de sentido. Reconocemos, entonces, en lo que relata esta estudiante, una forma de exclusión, que actúa mediante la anticipación de que no se conseguirá el acceso a otro nivel del sistema. Esta anticipación liga el origen cultural y social de los estudiantes a su destino escolar y social: para tales grupos, tales destinos. Dar por sentado que los jóvenes indígenas no podrán seguir una carrera universitaria tiene como trasfondo una concepción naturalista y esencialista del éxito o el fracaso en la escuela (Rodríguez y Sulca, 2020). De este modo, se profundizan las diferencias. En otra parte de la entrevista, Sara plantea: "es como que los docentes nunca se preocuparon de que aprendamos cosas más lindas o cosas que nos puedan ayudar en una carrera universitaria, porque nunca pensaron que chicos de las comunidades quisieran estudiar una carrera [universitaria]" (Entrevista a Sara, extutora del ProTconPO, proveniente de una comunidad wichí).

Aquí, además, se incluye una cuestión de decisión: Sara siente que sus docentes nunca pensaron que ellos pudieran aspirar a seguir una carrera universitaria. Así aparece la idea de que en el horizonte de intereses de los indígenas no podría estar el ingreso al nivel universitario. A esto se suma el imaginario de que estos no aprenden de la misma forma o la idea de que no pueden aprender algunas cosas: 
había una profesora que era de Lengua y Literatura, era, son muy estrictas las profes de lengua, eso ya lo sé más o menos. Ella decía que no... que todos los chicos que estaban estudiando, en ese momento, eran como que no había manera de hacerles entender en sus clases, porque no entendían palabras, ni siquiera sabían usar diccionario, decía ella. (Entrevista a Sara, extutora del ProTconPO, proveniente de una comunidad wichí)

Novaro (2005) plantea que, en ocasiones, la diversidad es desvirtuada, y utilizada para atribuir todos los problemas o dificultades de los estudiantes a la cultura. Evidenciamos en el relato de Sara la tendencia por parte de esta docente a considerar la cultura como un obstáculo insuperable para el aprendizaje de su materia. Al respecto, Sara agrega que la docente "en parte tenía razón" porque la mayoría de sus compañeros no entendían el idioma, pero lo que resulta negativo para esta estudiante es que la respuesta de la docente fuera no enseñarles nada. Las consecuencias prácticas de estas representaciones y los discursos escolares derivan en "límites para enseñar" porque se supone que "no podrán aprender". De allí que la desigualdad social que afecta a los pueblos indígenas se traslada a la escuela y esta se vuelve, una vez más, funcional a la perpetuación de un orden social (Rodríguez y Sulca, 2020).

Si bien esta estudiante accedió a la universidad, las consecuencias de no haber accedido a conocimientos esenciales para su continuidad en el sistema educativo conducen a que el tránsito por el nivel universitario sea dificultoso, prolongado, y pueden llevar incluso a la deserción:

venir a la universidad era como que volver a empezar de nuevo, en tema de los estudios. Ahí me di cuenta que no aprendí nada en la secundaria, fue más difícil de lo que imaginaba. Hubo un momento en el que dije 'hasta acá nomás llegué y me vuelvo' porque era difícil (Entrevista a Sara, extutora del ProTconPO, proveniente de una comunidad wichí).

Lo que relata Sara es común a muchos de los integrantes de CEUPO: sentir que no son capaces de continuar con sus carreras porque están en desventaja respecto de quienes tuvieron mejores posibilidades educativas en el nivel secundario. De este modo, sostenemos que la educación secundaria ya no excluye explícitamente a los estudiantes indígenas -lo que se evidencia mediante su obligatoriedad-, pero sí existen aún formas de exclusión más sutiles, expresadas mediante la repitencia, el abandono -por ejemplo-, o mediante los discursos de algunos docentes que, en muchas ocasiones, convencen a los jóvenes de no continuar en el sistema.

Marta, por su parte, tuvo una experiencia muy diferente. En su colegio, la mayoría de los docentes eran de la misma comunidad; en sus palabras: "uno que otro profesor sería, capaz, de acá de capital o de... tenía profesores de Moldes, por ejemplo, pero no, eran varios que habían venido a estudiar y habían vuelto a trabajar de nuevo a Iruya" (Entrevista a Marta, participante del ProTconPO, proveniente de una comunidad kolla).

Por ello, en una situación opuesta a lo que relató Sara, el acceso de Marta a la universidad fue consecuencia de la iniciativa de una docente del colegio, quien llevó y repartió entre los estudiantes folletos informativos acerca de las carreras que ofrecía la UNSa.

En este sentido, mientras muchos docentes consideran inimaginable que un joven indígena pueda y quiera estudiar en la universidad, existen quienes 
alientan a estos jóvenes a imaginarlo y concretarlo. De esta manera, las instituciones educativas que, en el marco de la legislación vigente, asumen el compromiso con la educación de los pueblos indígenas desde una perspectiva respetuosa de sus culturas, también generan incertidumbre e indecisión en las trayectorias posibles o deseables para las niñas, los niños y los jóvenes indígenas.

\section{Continuidades, diferencias y nuevas experiencias: el ingreso a la universidad}

Con estos caminos recorridos, pese a todas las dificultades que encuentran en su trayectoria y a la falta de políticas que atiendan a la diversidad cultural desde una perspectiva intercultural, existen jóvenes indígenas que logran transitar por los diferentes niveles de educación formal y acceder a la Universidad. Tal como plantea Santana Colin (2018), esta llegada no es fortuita, sino consecuencia de una larga historia de luchas de los propios pueblos por el acceso a la educación. Asimismo, el ingreso a la universidad implica la apertura de una serie de negociaciones entre las y los jóvenes indígenas, las instituciones escogidas para sus familias y sus comunidades (Ossola, 2020).

Como hemos visto, muchos de estos estudiantes llegan tras años de discriminación en el sistema formal. De hecho, cuando los integrantes del CEUPO son consultados acerca de por qué creen que hay estudiantes indígenas de la UNSa que no se acercan a participar del ProTconPO, una respuesta unánime es que quizás prefieren que no se sepa que son indígenas; "pasar desapercibidos", para no sufrir nuevamente la discriminación. Algunos incluso nos cuentan anécdotas de estudiantes que provienen de su misma comunidad pero prefieren no decirles a sus compañeros y profesores que son indígenas $\mathrm{y}$, como formar parte del CEUPO implica exponerlo, temen enfrentarse a esa discriminación de la que fueron objeto durante años de escolarización:

Sí, muchos piensan que los van a discriminar, yo conozco chicos de mi pago que estudian aquí, en Ingeniería y en Económicas, pero ellos dicen que no van a decir que son de pueblo porque no quieren que los miren raro o los traten mal. (pasaje de conversación con un integrante del CEUPO, tomada de notas de campo)

También encontramos en algunos de los discursos la idea de evitar la lástima; por ejemplo, Sara nos contaba que en un principio ella no quería participar de estos espacios porque "era todo el tiempo de pueblos originarios, pueblos originarios, que hay que ayudarlos, y bueno, era como que sentía que había como lástima de por medio" (Entrevista a Sara, extutora del ProTconPO, proveniente de una comunidad wichí). Pero cuando se acercó se dio cuenta de que es un espacio importante para escuchar las voces de los propios indígenas.

Estos comentarios -sobre los motivos por los que algunos estudiantes indígenas no quieren que los identifiquen como tales, aun autorreconociéndose en esa identidad- vienen acompañados en los relatos de los integrantes del CEUPO por la importancia de continuar con estos espacios para las futuras generaciones. Es decir, para que los próximos estudiantes no sientan vergüenza ni miedo de dar a conocer su origen y su identidad indígena, porque en la UNSa ya está asumido que existe una Comunidad de Estudiantes de Pueblos Originarios, cuyas culturas merecen respeto. Así, encontramos en los integrantes del CEUPO lo que Soto, Luján y Rosso (2019) denominan un rol de "pioneros" universitarios, es decir, quienes realizan los primeros pasos en 
la construcción del CEUPO, abriendo camino para futuros estudiantes indígenas en la UNSa.

En línea con esto, los integrantes del ProTconPO recuperan como un logro el avance en relación con que la comunidad universitaria conozca cada vez más la existencia del CEUPO. Al respecto, Nilda expresa:

el CEUPO ganó visibilidad en la universidad, que se sabe que a la Universidad ingresan año a año estudiantes de pueblos originarios. Creo que esa es una de las grandes fortalezas, que posibilitan que también desde las mismas autoridades se preste atención a las inquietudes que el grupo y los chicos pueden tener. (Entrevista a Nilda, colaboradora y extutora del ProTconPO).

Esta cita nos permite identificar otro valor que se le atribuye a la visibilización, como posibilitadora de mayor apoyo por parte de las autoridades a estos espacios. Por su parte, José reflexiona:

yo ahora creo, desde que yo entré, a ver, desde que yo conozco el CEUPO y todos estos años pude ver que se está conociendo más el CEUPO, con las actividades, con todo, me parece que ya la Universidad sabe que hay estudiantes acá, que vienen de comunidades. (Entrevista a José, integrante del ProTconPO)

De igual manera, refieren a esto Sara y Marta, además de ser un comentario recurrente en los encuentros y talleres de los que participamos.

Con relación a lo que venimos desarrollando, algo particular sucede con los estudiantes indígenas de la Facultad de Ingeniería, que estudian en una unidad académica desde la cual se niega la presencia de indígenas. En esta facultad no convocan a concurso para tutor del ProTconPO, porque sostienen que no hay estudiantes indígenas: "nos pasa con Ingeniería, que las autoridades [...] dijeron 'nosotros no tenemos ningún chico' y nosotros sabemos que sí hay, no quisieron el tutor" (Entrevista a coordinadora del ProTconPO). Resuena en esta representación el imaginario de la Argentina compuesta exclusivamente por descendientes de europeos, negando la diversidad cultural y lingüística.

Sin embargo, pudimos identificar que asisten a esa facultad estudiantes indígenas, lo cual se corrobora cuando algunos de ellos se acercan al ProTconPO mediante otras facultades:

varias veces me pasó de que chicos de Ingeniería vinieron: 'me contaron que acá se trabaja también con Pueblos' así con timidez ¿no? 'con temas de Pueblos'... así han llegado un montón de chicos de Ingeniería a Exactas. 'Mirá vos', le digo (risas), yyo por dentro como... (Entrevista a Nilda, colaboradora y extutora del ProTconPO).

Por su parte, Marta tiene una hermana que estudia en Ingeniería y comenta que la invita a participar, pero ella no se siente cómoda porque no hay tutor de su facultad y tampoco otros estudiantes. Así, las decisiones de las autoridades de la facultad terminan truncando esfuerzos institucionales por incluir a estudiantes indígenas. Nilda también comenta que la mayoría de esos estudiantes no mantienen su participación en los espacios, por el hecho de no tener tutor de su propia facultad, pero además porque deciden no hacer visible su pertenencia cultural. Aquí se evidencia cómo esas representaciones son constitutivas de subjetividad, y contribuyen a que se niegue la identidad indígena. En este caso, existe una continuidad de esa idea que encontrábamos en las experiencias del secundario respecto de que los indígenas no llegan a la universidad. 
Se encuentran también diferencias al respecto, de hecho, que la UNSa desarrolle estrategias de retención como el ProTconPO ya representa un avance en la superación de la idea mencionada. Sin embargo, también hay esfuerzos en el mismo sentido de actores que no necesariamente se encuentran articulados con el ProTconPO. Por ejemplo, Sara resalta entre sus apoyos en la carrera a los docentes de primer año: "Siempre sentí ese apoyo de parte de todos los profes de primer año de la carrera. Es más, me conozco a todos mis profes de primer año y siempre los recuerdo con mucho cariño" (Entrevista a Sara, extutora del ProTconPO, proveniente de una comunidad wichí).

Allí, esta estudiante reconoce un sostén por parte de los docentes, que además se torna más valioso dado que, como vimos en páginas anteriores, nunca había experimentado algo similar en su trayectoria escolar. También en nuestra práctica docente en la UNSa (carreras de Antropología y Ciencias de la Educación), hemos notado de qué manera durante el primer año ciertas cátedras ofrecen importantes espacios de diálogo y conocimiento mutuo, los cuales ocurren en simultáneo con las propuestas del CEUPO y el ProTconPO.

Sin embargo, este aspecto positivo no se generaliza, justamente por obstáculos institucionales. Sara llegó a la UNSa como wichí, y todos sus docentes de primer año sabían que ella pertenecía a una comunidad indígena. Desde el ProTconPO, una de las estrategias de retención consiste en identificar a los ingresantes indígenas -mediante la encuesta aplicada en el Ciclo de Ingreso Universitario (CIU) - y luego informar a los docentes de primer año quiénes son. Pero este trabajo excede al tutor indígena de cada facultad, que difícilmente conozca a todos los docentes, por lo que se realiza desde los servicios de orientación y tutoría con los que cuenta cada una de ellas.

En este punto, entran en juego las diferencias en las relaciones de los tutores con los servicios de cada facultad, en tanto no en todos los casos la predisposición para articular con el tutor indígena es la misma y, en consecuencia, en algunas de ellas este trabajo no logra concretarse. Esto se traduce en que ese apoyo de los docentes de primer año se encuentre coartado por el desconocimiento de la pertenencia indígena de sus estudiantes. Según pudimos saber, solo en las Facultades de Ciencias de la Salud y de Ciencias Exactas, el tutor indígena se encuentra trabajando como parte de los equipos de orientación de esas unidades académicas. A pesar de estos obstáculos, el apoyo brindado por los docentes de primer año y del CIU es considerado una valiosa estrategia para la retención. Así, todos los tutores reconocen en estos la mayor apertura al trabajo con estudiantes indígenas, respeto y disponibilidad, aun cuando a muchos la situación de tener indígenas en sus aulas les despierte inseguridades y miedos:

$Y$ de a poco los profes [de primer año] fueron dando una mano y ellos también inquietos ¿no? Porque dice ‘¿y qué hago yo con uno de pueblos originarios?' es una así ‘¿pero qué? ¿me va a entender?' y le digo 'profe es un alumno, es un estudiante, como todos, usa un celular como usted'. (Entrevista a Nilda, colaboradora y extutora del ProTconPO)

Además de describir estos miedos e inseguridades, este pasaje permite identificar la imagen de los indígenas que tienen algunos docentes, como si todos conservaran su lengua indígena, cuando en realidad la mayoría de ellos no pudieron hacerlo. Esta representación nos lleva a plantear la actualización de una temática abordada anteriormente: la clasificación dominante en el imaginario salteño, que ubica a los estudiantes kolla, por ejemplo, en una categoría 
diferente a la de los estudiantes wichí (Ossola, 2015). En tanto estos últimos representan mejor esa imagen arcaica del indígena, principalmente por dos aspectos: la ubicación geográfica de sus comunidades - generalmente alejadas de las ciudades- y la conservación de la lengua. Sin embargo, la desigualdad socioeconómica y el racismo es común a todos los sectores indígenas (Ossola, 2015).

El reconocimiento de la diversidad en la diversidad se constituye en otra de las temáticas trabajadas desde el ProTconPO y el CEUPO. De este modo, se busca evidenciar hacia el interior del grupo - pero también en actividades para el resto de la comunidad- cómo se tornó necesaria la migración, de forma tal que muchos indígenas abandonaron sus comunidades incluso antes de poder ingresar a la Universidad. Asimismo, se valorizan las lenguas indígenas, a la vez que se hace énfasis en lo difícil que es sostener su vitalidad, en algunos casos, y recuperarlas, en otros. Conocer las realidades de cada uno y compartir la cotidianeidad constituyen estrategias que permiten crear lazos fuertes que sostengan la permanencia. Así, por ejemplo, una de las estudiantes comenta que abandonó muchas veces sus estudios, porque ya tiene hijos y trabaja, pero que siempre siguió en contacto con el CEUPO y por eso retomó la carrera y está pronta a recibirse.

No queremos cerrar este artículo sin antes referirnos a dos cuestiones con las que se encuentran los estudiantes indígenas en la universidad, que la mayoría de ellos no experimentaron en niveles anteriores de escolaridad:

Por un lado, el cambio en la organización burocrática y del tiempo. Si bien en los otros niveles muchas veces se les llamaba la atención por no asistir a las instituciones debido a festividades o actividades propias de su cultura, en el caso de la UNSa, se encuentran con consecuencias fuertes al respecto:

con uno de los chicos de Química en Exactas, que él es de Iruya, el no bajaba de Iruya en cierta época, no rendía en febrero-marzo, porque es época de las cosechas y del carnaval, y tenía la beca Bicentenario... eh... la pierde a la beca Bicentenario por no rendir en esas fechas, justo porque tenía que cumplir con las dos materias que le exigían. (Entrevista a Nilda, colaboradora y extutora del ProTconPO).

La administración del tiempo para el estudio y para la realización de otras actividades -principalmente ligadas al ámbito comunitario- son temas de reflexión y debate en los espacios de tutoría. Uno de los ejes para entender las formas de habitar la universidad por parte de los estudiantes indígenas es comprender la complementariedad entre las actividades que se realizan en la ciudad (entre ellas, el estudio en la universidad) y las que se desarrollan en la comunidad.

El acompañamiento en los trámites burocráticos constituye también una estrategia de retención. Así, desde el ProTconPO se acompañó a este estudiante en todo el proceso para realizar el reclamo correspondiente y fundamentado. Finalmente, la beca le fue restituida. Se acompaña, de este modo, a todos los estudiantes del CEUPO en aquellos trámites que desconocen, hasta tanto adquieren independencia al respecto.

Por otro lado, se produce el encuentro con la lectura académica universitaria, que presenta grandes dificultades para el estudiantado en general, pero más aún para los indígenas. Al respecto, Sara expresaba: 
pero sí me dificultaba mucho el tema de los materiales, de los autores, porque había palabras que nunca había escuchado, ehm... y bueno, fui aprendiendo, fui... fui... perdiendo mi miedo y también de ir preguntando, usar el diccionario y buscar palabras que me parecían desconocidas. (Entrevista a Sara, extutora del ProTconPO, proveniente de una comunidad wichí)

$\mathrm{Al}$ respecto, Santana Colin (2018) sostiene que el lenguaje académico es una de las herramientas más valiosas que adquieren los indígenas que logran acceder a la universidad, porque luego les permite manifestar en ese mismo código sus preocupaciones y las del mundo indígena. Sin embargo, los materiales universitarios en muchas ocasiones se constituyen en límites para el aprendizaje de estos estudiantes. Nilda también nos habló al respecto:

Es un trabajo muy abstracto y es un triple o cuatro veces mayor el esfuerzo que tenés que hacer para comprender un texto, para leer un texto, leer una página de un texto. Entonces es un trabajo muy individual, agotador diría yo, cansador y hasta frustrante para el estudiante. (Entrevista a Nilda, colaboradora y extutora del ProTconPO)

Nos interesa focalizar en ese último comentario, en la frustración que produce el hecho de no entender lo que leen. El acompañamiento en estos procesos de lectura, de forma individual y grupal, es una de las estrategias de retención que se desarrollan desde el ProTconPO. Dadas las singularidades que tienen la enseñanza y el aprendizaje en contextos de diversidad cultural y lingüística, en este tipo de situaciones son replanteadas las formas de compartir conocimientos. Así pues, deben deconstruirse las ideas previas en torno a un concepto académico, las maneras y los estilos del acompañamiento tutorial, y la mirada propia en torno de qué es aprender en la universidad.

\section{A modo de cierre}

Si bien la Universidad es considerada una de las instituciones más representativas de la colonialidad-modernidad, en el caso de la UNSa reconocemos que se tornan posibles experiencias que posibilitan disputas de sentidos. Emergen esfuerzos por permear los límites que impone este modelo y la apertura de la institución permite que esos se constituyan en intersticios. De esta manera, estudiantes que han desarrollado trayectorias educativas de invisibilización de su identidad indígena, encuentran en la UNSa un lugar donde posicionarse política y epistémicamente desde la idea de hacer evidente su identificación étnica.

Encontramos, en este breve recorrido por las trayectorias educativas de los estudiantes del CEUPO, dos huellas principales, que influyen en las estrategias de permanencia que despliegan estos jóvenes.

Por un lado, la negación de lo indígena como tal, representada en la idea de lo indígena como parte del pasado, en la negativa a hacer presentes en la institución educativa aspectos característicos de su cultura, como el idioma, entre otros aspectos.

Por otro lado, la negación de que los indígenas puedan llegar a la Universidad, evidente en los discursos de algunos docentes del nivel secundario y de algunas autoridades universitarias, como es el caso de la Facultad de Ingeniería. 
La respuesta a estas representaciones es la de (re)marcar su presencia. La estrategia consiste entonces en deconstruir esas ideas a partir de un trabajo de hacerse visibles como estudiantes universitarios indígenas. No como estrategia individual, sino colectiva, para que futuros estudiantes no vivan esas negaciones y se instale en la comunidad universitaria que dentro de la UNSa existe una Comunidad de Estudiantes Universitarios de Pueblos Originarios, que ingresan, permanecen y se reciben en esa Universidad.

Sostenemos hacerse presentes y visibles como estudiantes universitarios indígenas es consecuencia de los estigmas mencionados y constituyen una estrategia para intentar superarlos. Por y para ellos, pero también de manera colectiva para los futuros estudiantes indígenas de la UNSa.

En esta línea, el egreso también constituye -además de ser el objetivo propio de cada estudiante- una estrategia de permanencia, en tanto muchos de los integrantes del CEUPO que han alcanzado su título continúan participando de las actividades. Seguir trabajando con el CEUPO representa una forma diferente de contribuir con la comunidad por parte de los graduados indígenas, en tanto apoyan a la formación universitaria de jóvenes indígenas, a la vez que muestran a los sectores citadinos que las culturas indígenas continúan vivas en Argentina. Entonces, este sostenimiento de los vínculos, luego del egreso, no es solo a fin de colaborar en las cuestiones académicas y afectivas, sino para demostrar, en aquellas actividades que tienen como destinataria a la comunidad universitaria en general, que hay graduados indígenas de la UNSa. Esto constituye así un "retorno", que no conlleva necesariamente el regreso físico a las comunidades (Czarny, Ossola y Paladino, 2018).

El análisis realizado permite reconocer que se van produciendo modificaciones identitarias en los diferentes niveles, relacionadas con el ocultamiento y reivindicación de la identidad indígena. En el caso del nivel universitario, la formación allí recibida -no solo en las carreras cursadas sino en otros ámbitos, incluidos el CEUPO y el ProTconPO- permite a muchos estudiantes indígenas reencontrarse con las identificaciones étnicas desde un espacio de reivindicación comunitaria.

\section{Financiamiento}

Consejo Nacional de Investigaciones Científicas y Técnicas. Proyecto Institucional “Territorialidad y poder. Conflictos, exclusión y resistencias en la construcción de la sociedad en Salta", del Instituto de Investigaciones en Ciencias Sociales y Humanidades (ICSOH), apoyo económico para trabajo de campo.

\section{Agradecimientos}

Este trabajo cuenta con el apoyo del Proyecto Institucional "Territorialidad y poder. Conflictos, exclusión y resistencias en la construcción de la sociedad en Salta” (ICSOH), del proyecto de investigación del Consejo de Investigación de la Universidad Nacional de Salta (CIUNSa) $N^{\circ} 2317 / 17$ y del Proyecto PICT N²825-2018. Se agradece especialmente a los miembros del proyecto de tutorías con los estudiantes de pueblos originarios de la UNSa. 


\section{Q Referencias bibliográficas}

" Aikman, S. (2003). La educación indígena en Sudamérica. Interculturalidad y bilingüismo en Madre de Dios, Perú. Lima:IEP Ediciones.

"Anaya, (2012). Informe del Relator Especial sobre los derechos de los pueblos indígenas, James Anaya. La situación de los pueblos indígenas en Argentina. Ginebra: Naciones Unidas.

"Czarny, G. (2008). Pasar por la escuela: Indígenas y procesos de escolaridad en la ciudad de México. México: Universidad Pedagógica Nacional.

»Czarny, G., Ossola, M. y Paladino, M. (2018). Presentación. Dossier Jóvenes indígenas y universidades en América Latina: sentidos de la escolaridad, diversidad de experiencias y retos de la profesionalización. Antropología Andina Muhunik - Jathasa, 5(1), 6-17. Recuperado de: http://revistas.unap.edu.pe/antroa/index.php/ANTRO/article/view/320/297

》Delory-Momberger, C. (2009). Biografía y educación: figuras del individuo-proyecto. Ciudad Autónoma de Buenos Aires: Editorial de la Facultad de Filosofía y Letras UBA.

» Hirsch S. y Serrudo, A. (2010). La educación en comunidades indígenas de la Argentina: de la integración a la Educación Intercultural Bilingüe. En S. Hirsch y A. Serrudo (Comps.). La educación Intercultural Bilingüe en Argentina. Identidades, lenguas y protagonistas. Pp. 17-44. Buenos Aires: Noveduc.

»Instituto Nacional de Estadísticas y Censos (INDEC) (2004-2005). Encuesta Complementaria de Pueblos Indígenas (ECPI). Buenos Aires: INDEC.

"Instituto Nacional de Estadísticas y Censos (INDEC) (2010). Censo Nacional de Población, Hogares y Viviendas 2010. República Argentina. Buenos Aires: INDEC.

"Levinson, B., Sandoval Flores, E. y Bertely-Busquets, M. (2007). Etnografía de la educación. Tendencias actuales. Revista Mexicana de Investigación Educativa, 12(34), 825-840.

" Luján, A., Soto, M. y Rosso, L. (2018). Experiencias de ingreso y permanencia de estudiantes indígenas en una universidad convencional del nordeste Argentino. Antropología Andina Muhunchik - Jathasa 4(1). Recuperado de: http://revistas.unap.edu.pe/antroa/index.php/ANTRO/article/view/322

"Novaro, G. (2005). Representaciones Docentes sobre las “Formas de Socialización” y las Posibilidades de Aprendizaje de los Alumnos Indígenas. Campos Revista de Antropología Social, 6 (1-2), 79-95.

» Ossola, M. M. (2015). Aprender de las dos ciencias. Etnografía con jóvenes Wichí en la educación superior de Salta. Santiago del Estero: EDUNSE.

» Ossola, M. (2020). Jóvenes indígenas y educación superior en Argentina. Experiencias y desafíos del ingreso, la permanencia y la graduación universitaria. Desidades, 26(8), 39-51. Recuperado de: http://desidades.ufrj.br/es/featured_topic/jovenes-indigenas-yeducacion-superior-en-argentina-experiencias-ydesafios-del-ingreso-la-permanenciay-la-graduacion-universitaria/

" Ossola, M. M. y Hecht, A. C. (2011). Relatos de jóvenes sobre el Wichí y el español en sus trayectorias escolares en la provincia de Salta. Novedades Educativas, 23(244), 7-11.

"Rodríguez y Sulca (2020). El acceso a la educación y la inclusión socioeducativa de estudiantes indígenas. Provincia de Salta-Argentina. Textura - Revista de Educação e Letras, 22(51), 194-218. doi: https://doi.org/10.17648/textura-2358-0801-v22n51-5683 
"Rodríguez, N. (2020). Interculturalidad y Universidad: Desarrollo, avances y perspectivas del Proyecto de Tutorías con Estudiantes de Pueblos Originarios en la Universidad Nacional de Salta (Tesis de doctorado). Facultad de Filosofía y Letras, Universidad Nacional de Tucumán, Tucumán, Argentina.

» Santana Colin, Y. (2018). Formación académica y militancia de los intelectuales indígenas mexicanos. Antropología Andina Muhunchik - Jathasa, 4(1). 79-102.

»Soto, M. G., Luján, A. E. y Rosso, L. L. (2019). Nuevas lecturas a la permanencia de estudiantes indígenas en una Universidad Convencional argentina. Ponencia presentada en el zer Congreso Internacional de Pueblos Indígenas de América Latina. Brasilia, 3-5 julio.

» Sulca, E. (2018). Autobiografía y subjetividad. Significados de las experiencias escolares desde mi ser indígena. Actas de las IX Jornadas de Investigación en Antropología Social Santiago Wallace. Buenos Aires: UBA.

» Terigi, F. (2006). Tres problemas para las políticas docentes. Actas del II Encuentro Internacional: La docencia, zuna profesión en riesgo? Montevideo: OREALC. http://miguelricci2008.blogspot.com.ar/2010/og/tres-problemas-para-las-politicas.html

"Terigi, F. (2008). En la perspectiva de las trayectorias escolares. Comentario al capítulo 3 del Atlas de las desigualdades educativas en América Latina: La asistencia escolar en la actualidad. trayectorias educativas en 8 países de América Latina. Buenos Aires: SITEAL IIPE - UNESCO Sede Regional Buenos Aires.

» UNICEF (Fondo de la Naciones Unidas para la Infancia) (2009). Los pueblos indígenas en Argentina y el derecho a la educación. Situación socioeducativa de niñas, niños y adolescentes de comunidades rurales wichí y mbyá guaraní. Buenos Aires: UNICEF. 